\title{
The Structure, Stability and Reactivity of Mo-oxo species in H-ZSM5 Zeolites: Density Functional Theory Study
}

Danhong Zhou ${ }^{1,2}$, Yuan Zhang ${ }^{1}$, Hongyuan Zhu ${ }^{1}$, Ding $\mathrm{Ma}^{2}$, Xinihe Bao ${ }^{* 2}$

${ }^{1}$ Institute of Chemistry for Functionalized Materials, College of Chemistry and Chemical Engineering, Liaoning Normal University, Dalian 116029, People’s Republic China

${ }^{2}$ State Key Laboratory of Catalysis, Dalian Institute of Chemical Physics, Chinese Academy of Science, 457 Zhongshan Road, Dalian 116023, People's Republic China

\section{Supporting materials}


TABLE S1: The Geometric Parameters of $\mathrm{Mo}_{2} \mathrm{O}_{5}{ }^{2+}$ Dimer Located at Different Adjacent Brönsted Acidic Sites. Optimization were Performed at BLYP/DNP Level with 6T (10T) Cluster Model (Bond Length in Å, Bond Angle in Degree)

\begin{tabular}{|c|c|c|c|c|c|c|c|}
\hline & T6T6 & T6T9 & T3T3 & T3T12 & T7T12-R & T7T12-L & Experimental Data \\
\hline $\mathrm{Mo} \equiv \mathrm{O}$ & 1.733 & 1.731 & 1.733 & 1.735 & 1.734 & 1.726 & $\begin{array}{l}\mathrm{Mo}=\mathrm{O} \text { double bond: } \\
1.69 \pm 0.008 ;{ }^{12}\end{array}$ \\
\hline $\mathrm{Mo} \equiv \mathrm{O}$ & 1.732 & 1.731 & 1.732 & 1.731 & 1.727 & 1.738 & $1.73 ;{ }^{53}$ \\
\hline $\mathrm{Mo} \equiv \mathrm{O}$ & 1.733 & 1.734 & 1.733 & 1.735 & 1.735 & 1.729 & Mo-O single bond: \\
\hline $\mathrm{Mo} \equiv \mathrm{O}$ & 1.732 & 1.731 & 1.732 & 1.731 & 1.728 & 1.736 & $1.78 \pm 0.0009 ;{ }^{12,53}$ \\
\hline Mo-O & 1.926 & 1.888 & 1.928 & 1.886 & 1.939 & 1.944 & Mo-O single bond in \\
\hline Mo-O & 1.926 & 1.974 & 1.928 & 1.975 & 1.939 & 1.936 & -Mo: \\
\hline Мo--Mo & 3.570 & 3.553 & 3.554 & 3.523 & 3.500 & 3.477 & $1.84 \pm 0.0009 ;{ }^{12,51}$ \\
\hline $\mathrm{Mo}-\mathrm{O}_{\mathrm{F}}$ & 2.158 & 2.563 & 2.185 & 2.511 & 2.203 & 2.262 & Mo--Mo distance: \\
\hline $\mathrm{Mo}-\mathrm{O}_{\mathrm{F}}$ & 2.214 & 2.092 & 2.200 & 2.094 & 2.199 & 2.133 & $3.70 \pm 0.0003 ;{ }^{12}$ \\
\hline $\mathrm{Mo}-\mathrm{O}_{\mathrm{F}}$ & 2.214 & 2.135 & 2.201 & 2.130 & 2.165 & 2.116 & $\begin{array}{l}\text { Mo--Al distance: } 3.60 \\
\pm 0.0006{ }^{12}\end{array}$ \\
\hline $\mathrm{Mo}-\mathrm{O}_{\mathrm{F}}$ & 2.158 & 2.156 & 2.182 & 2.174 & 2.240 & 2.296 & \\
\hline Mo--Al & 3.065 & 3.298 & 3.055 & 3.118 & 3.096 & 3.107 & \\
\hline Mo--Al & 3.065 & 3.039 & 3.053 & 3.034 & 3.116 & 3.084 & \\
\hline $\mathrm{O}_{\mathrm{F}-\mathrm{O}} \mathrm{O}_{\mathrm{F}}$ & 4.340 & 4.613 & 4.344 & 4.624 & 4.023 & 4.092 & \\
\hline $\mathrm{Mo}-\mathrm{O}_{\mathrm{F}}-\mathrm{Al}$ & 99.9 & 114.6 & 100.0 & 112.0 & 101.6 & 100.3 & \\
\hline $\mathrm{Mo}-\mathrm{O}_{\mathrm{F}}-\mathrm{Al}$ & 99.9 & 102.2 & 100.0 & 102.1 & 102.9 & 103.7 & \\
\hline
\end{tabular}


TABLE S2: The potential energies of optimized reactant, transition state and product for methane $\mathrm{C}-\mathrm{H}$ bond dissociation. (at B3LYP/6-31G**/LanL2DZ level).

\begin{tabular}{ll}
\hline Model & $E_{\text {tot }} /$ a.u. \\
\hline $\mathrm{CH}_{4}$ & -40.52402 \\
Reactant & -3592.88784 \\
Transition state & -3633.31097 \\
Product & -3633.35126
\end{tabular}

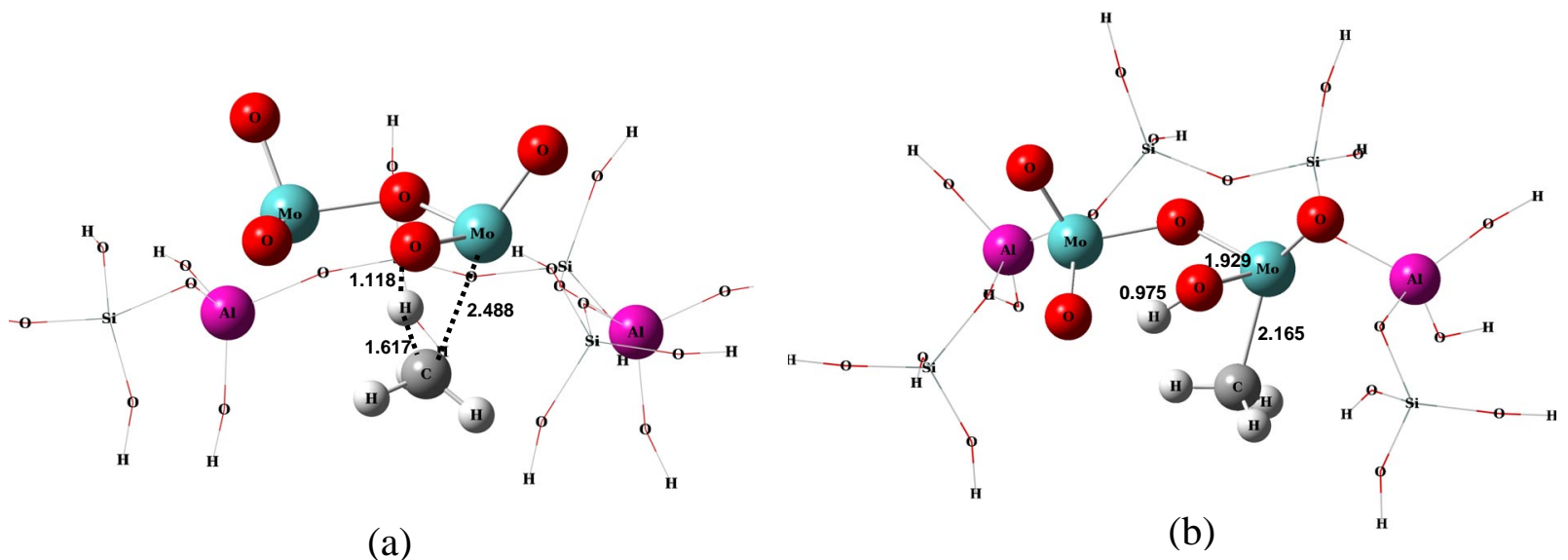

Figure S1. Optimized structure of transition state (a) and product (b) for methane C$\mathrm{H}$ bond dissociation. The selected distances are in $\AA$. 